\title{
Pour une meilleure appréhension de la variété des trajectoires d'internationalisation des entreprises artisanales
}

\author{
Thevenard-Puthod, Catherine \\ IREGE - Université Savoie Mont Blanc \\ Catherine.puthod@univ-smb.fr
}

\section{Résumé :}

Le champ de l'entrepreneuriat international s'est fortement développé ces 20 dernières années et s'élargit aujourd'hui à toute forme de comportements entrepreneuriaux à l'international. Toutefois, malgré cet élargissement, les start-up et les entreprises de secteurs high tech demeurent les objets centraux des recherches. Peu de travaux s'intéressent aux entreprises de petite taille non récemment créées et appartenant à des activités traditionnelles, telles les entreprises artisanales (EA). L'objectif de cet article est donc d'améliorer la connaissance sur les processus utilisés par les entreprises artisanales pour se développer à l'international et sur les facteurs internes et externes qui influencent ces processus. Etant donné le caractère exploratoire de cette recherche, nous avons utilisé une méthodologie qualitative fondée sur l'étude de sept cas d'EA s'étant internationalisées. Le matériau empirique collecté permet d'identifier l'existence de différents types de trajectoires d'internationalisation au sein des EA. Il met également en avant le rôle plusieurs facteurs internes (la rareté du savoir-faire, le caractère innovant des produits ou services proposés et l'orientation entrepreneuriale du dirigeant) dans l'adoption des processus les plus rapides et précoces

Mots-clés : Entreprise artisanale - Internationalisation - Entrepreneuriat international Savoir-faire - Profil du dirigeant 


\section{Quelles trajectoires d'internationalisation pour l'entreprise artisanale?}

\section{INTRODUCTION}

Le champ de l'entrepreneuriat international s'est fortement développé ces 20 dernières années (Servantie et al, 2016). Au départ plutôt axé sur les entreprises en création, et plus particulièrement sur les born global ou les International New Ventures (Madsen et Servais, 1997 ; Jones et al, 2011), il s'élargit aujourd'hui à toute forme de comportements entrepreneuriaux à l'international. Ainsi Oviatt et McDougall (2005, p.538) définissent l'entrepreneuriat international comme «la découverte, l'engagement, l'évaluation et l'exploitation d'opportunités, en dehors des frontières nationales (...)». Toutefois, malgré cet élargissement du champ, les start-up demeurent un objet central d'étude (Burgel et Murray, 2000 ; Anderson et al, 2004 ; Cavusgil et Knight, 2015) et très peu de travaux s'intéressent aux entreprises de petite taille non récemment créées. En outre, les entreprises nouvelles étudiées sont la plupart du temps issues des secteurs high tech (Bell, 1995 ; Coviello et Munro, 1997 ; Jones, 1999). Il n'existe quasiment aucune recherche sur les entreprises plus traditionnelles, comme les entreprises artisanales (EA), qu'elles soient récentes ou non. Paradoxalement, alors que l'artisanat a un poids économique important dans la plupart des pays développés (Paige et Littrell, 2002 ; Jaeger, 2009) et bien que les études statistiques disponibles montrent une réelle activité à $1^{1}$ export $^{1}$ de ces entreprises ${ }^{2}$, leurs stratégies d'expansion internationale demeurent peu explorées. Ceci tient certes au fait que les définitions de l'artisanat diffèrent selon les pays $^{3}$, mais surtout à la persistance de

\footnotetext{
${ }^{1}$ Le taux d'exportation des PME est considéré comme un critère pertinent pour mesurer l'engagement actif des entreprises dans les processus d'internationalisation. De nombreuses études montrent, en effet, que l'exportation permet l'entrée à faible coût et à faible risque sur les marchés étrangers et demeure ainsi le vecteur privilégié de l'internationalisation des PME (Lu et Beamish, 2001).

2 On estime en effet à 35000 le nombre d'entreprises artisanales françaises qui exportent leurs produits ou services, soit $6 \%$ du tissu artisanal. Ce secteur représente ainsi près d'un exportateur sur trois en France (ISM, 2012). BPI France (2014) indique par ailleurs que ce sont les TPE qui tirent à la hausse le nombre d'entreprises exportatrices en France. Le chiffre d'affaire réalisé par ces entreprises de petite taille à l'export est certes faible, puisqu'il s'élevait à 14,4 milliards d'euros en 2012, soit seulement 3\% du total réalisé par les entreprises françaises, mais ce chiffre est en régulière augmentation.

${ }^{3}$ En France, une entreprise artisanale est une personne physique ou morale exerçant à titre principal ou secondaire une activité professionnelle indépendante de production, de transformation, de réparation ou de prestation de services figurant sur une liste établie par décret en Conseil d'État. À côté de l'activité de l'entreprise, un second critère définissait l'entreprise artisanale : celle-ci ne devait initialement pas dépasser le
} 
représentations portant sur les EA (Paturel et Richomme-Huet, 2007 ; Thévenard-Puthod et Picard, 2015). On considère ainsi souvent que leur faible niveau de ressources et compétences et leur tendance à la myopie vis-à-vis de leur environnement (Torrès, 2003 ; Pacitto et Julien, 2006 ; Picard, 2006) les poussent à limiter leur champ d'action au marché local (Boldrini et al, 2011). Certains considèrent également que, compte tenu des coûts et des compétences nécessaires pour exporter, une entreprise de trop petite taille éprouvera des difficultés pour initier et surtout maintenir une stratégie d'internationalisation (Majocchi, et al, 2005).

Compte tenu du peu de connaissances disponibles sur les pratiques d'internationalisation des entreprises artisanales (EA) et des enjeux liés à la réussite de leur développement à l'étranger, cet article amène des réponses à deux questions de recherche : quelles sont les trajectoires d'internationalisation des EA ? Quels sont les facteurs explicatifs du déroulement de ces trajectoires ? Etant donné le caractère exploratoire de cette recherche, nous avons utilisé une méthodologie qualitative basée sur l'étude de sept cas d'EA s'étant internationalisées. Le matériau empirique collecté permet d'identifier l'existence de différents types de trajectoires d'internationalisation au sein des EA, plus ou moins rapides et précoces. Il met également en avant le rôle de plusieurs facteurs internes (la rareté du savoir-faire, le caractère innovant des produits ou services proposés et l'orientation entrepreneuriale du dirigeant) dans l'adoption des trajectoires les plus rapides et précoces. Cet ensemble de résultats vient alors enrichir les connaissances sur les stratégies d'internationalisation des entreprises artisanales, firmes $a$ priori différentes des start-up high tech largement étudiées par les précédents chercheurs, et alimente ainsi le champ de recherche de l'entrepreneuriat international.

La première partie détaille le cadre conceptuel de la recherche : la présentation des processus types d'internationalisation généralement décrits dans la littérature, puis la recension des principaux facteurs à même d'expliquer l'adoption de l'une ou l'autre de ces trajectoires. Elle se termine par l'explicitation de la méthodologie qualitative qui a guidé le recueil de données sur le terrain et présente les entreprises artisanales étudiées. Dans la seconde partie, nous présentons le matériau empirique collecté et les enseignements de cette recherche.

seuil de 10 salariés. Depuis la loi du 5 juillet 1996 instaurant un « droit de suite », cette limite de taille n'est plus un critère discriminant, puisque les entreprises qui le souhaitent peuvent rester immatriculées au répertoire des métiers au-delà de ce seuil. 


\section{1- CONSTITUTION DU CADRE D'ANALYSE PERMETTANT D'APPREHENDER LES PROCESSUS D'INTERNATIONALISATION DES EA}

A partir des connaissances disponibles dans la littérature sur l'entrepreneuriat international, il s'agit tout d'abord de construire un cadre d'analyse permettant d'appréhender les différents processus qu'une entreprise de petite taille peut suivre pour internationaliser son activité (§1.1). On identifie ensuite les principaux facteurs pouvant influencer l'adoption de ces processus $(\$ 1.2)$. On présente enfin la méthodologie qui a guidé le recueil et l'analyse des données $(\S 1.3)$.

\section{1/LES DIFFERENTS MODELES D'INTERNATIONALISATION DES ENTREPRISES}

Bien que le champ de l'entrepreneuriat international se soit surtout développé depuis la fin des années 90, de nombreux chercheurs se sont intéressés à l'internationalisation des PME dès les années 70. Les recherches se résument aujourd'hui à trois principaux points de vue sur ce processus.

Historiquement, les recherches entamées dans les pays nordiques ont donné lieu à la conception de modèles par étapes (Leonidou et Katsikeas, 1996), dont le plus connu est le modèle Uppsala (Johanson et Vahlne, 1977). Selon ce dernier, l'internationalisation consiste en un processus évolutif et continu, au cours duquel l'implication des entreprises à l'international augmente graduellement, en fonction de leurs connaissances des marchés et de leurs compétences internationales (Johanson et Vahlne, 1977). Ainsi les entreprises débutent leur internationalisation dans des pays à faibles distances psychiques (autrement dit, des pays où les différences de langages, de pratiques et de cultures sont limitées), avant d'attaquer des pays plus éloignés géographiquement et culturellement. Elles suivent également une « chaine d'établissement » traduisant des modes d'engagement de plus en plus poussés dans les pays étrangers. Ainsi l'internationalisation démarre en général avec une activité d'exportation, via un agent local indépendant ; puis grâce à l'accumulation des connaissances sur ce marché, l'entreprise met en place des filiales, d'abord commerciales, puis productives. Ce modèle met donc au cœur du développement international des entreprises les connaissances (Meier et Meschi, 2010), qu'elles soient générales (concernant la démarche d'internationalisation, les outils à utiliser...) ou spécifiques à un marché étranger (connaissance des clients, des canaux de distribution, des réglementations locales). 
Puis, à partir des années 90, des chercheurs issus de l'entrepreneuriat international ont mis à jour des processus d'internationalisation beaucoup plus rapides que ce modèle initial (Oviatt et McDougall, 1994 ; 2005). Ils ont identifié des entreprises qualifiées de «Born Global» qui semblent avoir un certain nombre de caractéristiques uniques : elles sont relativement jeunes (moins de trois ans d'existence en général), elles cherchent à répondre aux marchés internationaux dès leur création, et leurs revenus sont principalement générés par les marchés étrangers (au moins 25\% du C.A.; Madsen, et Servais, 1997). Les marchés géographiques sont choisis en fonction de leur potentialité et non en fonction de leur proximité avec le marché domestique. Elles appartiennent la plupart du temps à des secteurs d'activité à forte intensité de connaissances et de technologies et vendent des produits innovants (Oviatt et McDougall, 1994 ; Bell, 1995 ; Bloodgood, et al 1996). Du fait de leur haut niveau de connaissances, ces entreprises ont la capacité à s'adapter plus rapidement à un nouvel environnement. Elles bénéficient également de réseaux internationaux qui leur permettent d'avoir un meilleur accès à l'information et d'identifier plus rapidement les opportunités de développement. Pour ces chercheurs, les entreprises plus traditionnelles sont censées s'internationaliser plus lentement, en suivant des processus davantage proches des modèles gradualistes (Oviatt et McDougall, 2005; Rialp, et al 2005).

Ces deux premières visions du processus d'internationalisation diffèrent donc à la fois sur sa vitesse (progressif versus rapide) et sur la période à laquelle celui-ci démarre (précoce versus plus tardif), comme l'illustre le tableau 1. Toutefois, une troisième vision de ce processus est également apparue avec la «version 2 » du modèle d'Uppsala (Johanson et Valhne, 1990 ; Johanson et Vahlne, 2009). Dans cette version revisitée, les auteurs reconnaissent que l'insertion d'une entreprise dans des réseaux inter-organisationnels peut accélérer le processus d'internationalisation des PME, grâce aux connaissances et aux ressources financières, technologiques et commerciales apportées par des partenaires (fournisseurs, clients, distributeurs ; Chetty et Campbell-Hunt, 2003), facilitant ainsi l'accès aux marchés étrangers. De plus, la notion de distance psychique tend à s'atténuer, puisque le partenaire dispose déjà des connaissances sur le marché local. De même, d'autres chercheurs (Bell et al, 2003 ; Dominguez et Mayrhoffer, 2016) ont également mis à jour l'existence d'entreprises qualifiées de Born Again Global. Celles-ci affichent une focalisation initiale sur le marché domestique, mais suite à différents événements internes (acquisition de nouvelles ressources critiques, changement de dirigeant, innovation), elles procèdent à une internationalisation très rapide de leur activité. 


\section{$\underline{\text { Tableau } 1 \text { - Les modèles d'internationalisation dans la littérature }}$}

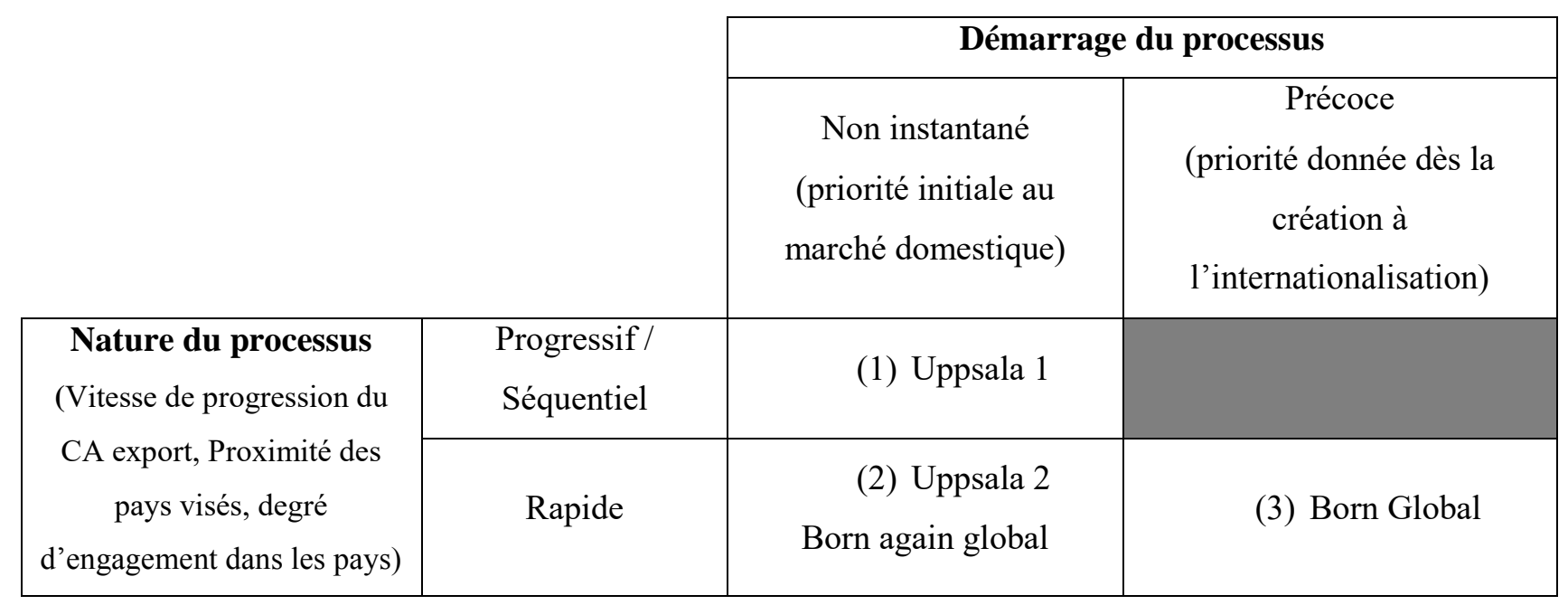

Aucun de ces travaux ne s'est intéressé au cas spécifique des EA. Toutefois, sur la base des travaux existants sur les caractéristiques de ces entreprises et les stratégies qu'elles empruntent, on pourrait a priori estimer que le processus qu'elles utilisent pour s'internationaliser risque d'être davantage proche du modèle Uppsala initial (quadrant $1 \mathrm{du}$ tableau). En effet, les EA sont traditionnellement considérées comme ayant des aspirations à la croissance limitées (Stanworth et Curran, 1976 ; Orser et al, 2000), pour ne pas dénaturer le savoir-faire (Polge, 2008 ; Boldrini et al, 2011), ni perdre le caractère informel et familial de l'organisation (Wiklund et al, 2003). La proximité géographique avec le marché et les différentes parties prenantes demeure également fondamentale (Loup, 2008; Torrès et Jaouen, 2008). Les EA semblent privilégier un rapport ancré dans la relation, peu instrumenté, où le contact physique avec la clientèle permet notamment de se différencier des entreprises de tailles plus importantes. Enfin, les dirigeants de ces entreprises sont plutôt caractérisés par une volonté farouche de conserver leur indépendance et de travailler seul (Zarca, 1986 ; Institut supérieur des métiers, 2010). Les entreprises qu'ils dirigent seraient donc moins insérées dans des réseaux que leurs homologues de plus grande taille (Jaouen, 2006). Cependant, compte tenu de l'hétérogénéité du monde artisanal (en termes de métiers, d'entreprises, de dirigeants... ; Institut Supérieur des Métiers, 2010), des mutations (pressions concurrentielles de plus en plus vives, mouvements de concentration, progrès technologique, 
arrivée de dirigeants aux nouveaux profils ${ }^{4}$ ) que connaissent ces secteurs d'activité et de l'existence de recherches récentes qui montrent que certaines EA peuvent opter pour des stratégies de croissance audacieuses (Thévenard-Puthod et Picard, 2015), on peut légitimement s'interroger sur la possibilité pour une entreprise artisanale d'adopter des processus d'internationalisation soit plus précoces, soit plus rapides, voire les deux. Cette interrogation se traduit dans l'énoncé de notre première question de recherche : quelle est la nature du processus d'internationalisation adopté par les EA ? Suivent-elles toujours un processus d'internationalisation progressif ou peut-on trouver en leur sein des born globals?

\section{2/ LES DETERMINANTS DU PROCESSUS D'INTERNATIONALISATION}

La littérature sur l'internationalisation des PME est vaste (Jean-Amans et Abdellatif, 2013) et il est difficile de proposer une revue exhaustive de tous les facteurs externes et internes explicatifs du processus. Nous avons ainsi retenu les cinq facteurs (trois externes, deux internes ; cf. tableau 2) qui étaient à la fois les plus couramment cités par les auteurs et qui nous paraissaient pertinents en contexte d'EA françaises ${ }^{5}$.

$\mathrm{Au}$ niveau des facteurs externes, on trouve la taille du marché domestique, le degré d'internationalisation des clients de l'entreprise et son insertion dans des réseaux.

- La taille du marché domestique est souvent considérée comme un facteur de précocité et de rapidité du processus d'internationalisation des PME (Oakey, 1995 ; Keeble, et al, 1998). On peut ainsi estimer que plus l'EA est sur un marché de niche, s'adressant seulement à quelques clients, plus elle va devoir s'internationaliser rapidement (Cabrol et Nlemvo, 2011).

- Certains chercheurs ont également souligné l'influence des clients sur le processus d'internationalisation des PME. Dans les activités B2B, la dépendance symbiotique (au sens de Pfeffer et Salancik, 1978) de certaines petites entreprises vis-à-vis de leurs

\footnotetext{
${ }^{4}$ En France pour les entreprises nouvellement créées ou reprises, 4 dirigeants sur 10 seraient aujourd'hui des anciens cadres ou professions intermédiaires et 50\% des dirigeants actuels auraient réalisé leur parcours professionnel en dehors de l'artisanat (Institut Supérieur des Métiers, 2010).

5 Par exemple, au niveau externe, certains auteurs (Laghzaoui, 2009) mentionnent l'influence du milieu internationalisant, c'est-à-dire l'ensemble des institutions et entreprises spécialisées pouvant faciliter le processus d'internationalisation. Notre étude ne portant que sur des entreprises artisanales françaises, elles bénéficient toutes de réseaux d'appui identiques (ex : Chambres de Métiers et de l'Artisanat, Business France...)
} 
clients donneurs d'ordre, les incite souvent à en suivre les exigences (Majkgard et Sharma, 1998 ; Lin et Chaney, 2007). Plusieurs recherches ont ainsi montré que les PME pouvaient être contraintes de suivre leurs clients à l'étranger, même lorsque ce développement à l'international n'était pas forcément planifié initialement (Holmlund et Kock, 1998 ; Ageron, 2001 ; Barbat et al 2014). Le type du client auquel une EA s'adresse peut donc influencer la vitesse ou la précocité avec laquelle elle va s'internationaliser. Si celle-ci travaille pour des clients français internationaux, elle pourra sans doute plus facilement se développer elle-même à l'international.

- Dans le même esprit, l'insertion de l'entreprise dans des réseaux d'affaires avec des clients ou des fournisseurs est souvent vue comme facilitant le démarrage du processus d'internationalisation (Ellis, 2000), grâce à l'apport d'informations ou d'opportunités sur les marchés étrangers (Coviello et Munro, 1997 ; Johanson et Vahlne, 2009). Ces réseaux sont également des facteurs d'accélération et de réussite du processus (Meier et Meschi, 2010). On pourrait donc estimer que l'expansion internationale de l'EA peut être facilitée par son insertion préalable dans des réseaux.

Les facteurs internes pouvant influencer le déroulement du processus d'internationalisation concernent le savoir-faire de l'entreprise artisanale et le profil de son dirigeant.

- Les entreprises artisanales se caractérisent par leur focalisation sur le métier (Picard, 2006 ; Polge, 2008), qui contient une part souvent importante de travail manuel et/ou de savoir-faire technique. On peut donc penser que le type de savoir-faire possédé va influencer le processus d'internationalisation; en particulier, son degré de rareté et le caractère innovant des produits développés. En effet, il a été reconnu dans la littérature que le degré d'innovation des produits proposés (Oviatt et McDougall, 1995), et plus largement la capacité d'innovation des PME, était un facteur facilitant l'internationalisation rapide de ces entreprises (Cassiman et Golovko, 2011 ; BeleskaSpasova, et al, 2012).

- Le rôle central du dirigeant dans la stratégie d'internationalisation des PME n'est enfin plus à démontrer (Coviello et Munro, 1995 ; Hutchinson et al, 2006 ; Ruzzier et al, 2007 ; Dominguez et Mayrhofer, 2016). On reconnait ainsi que ses compétences et son orientation internationales (sa compétence linguistique, sa connaissances des pays étrangers, sa maîtrise des techniques de l'export ... ; Westhead et al, 2001 ; Chetty et 
Campbell-Hunt, 2004 ; Fernandez-Ortiz et Lombardo, 2009...), qui peuvent être issues d'une formation préalable et/ou d'une expérience acquise à l'international (McDougall et al, 1994 ; Bloodgood et al, 1996 ; McDougall et al, 2003 ; Rialp et al., 2005 ; Hutchinson et al, 2006), influencent fortement l'intensité et la rapidité du développement à l'international. Plus globalement, son comportement entrepreneurial favoriserait également cet engagement (Barbat et al, 2014). Dans les entreprises artisanales, le pouvoir étant plus concentré que dans les PME, on peut penser que le profil du dirigeant va être encore plus déterminant (Miesenbock, 1988).

$\underline{\text { Tableau } 2 \text { - Les facteurs d'influence potentiels du processus d'internationalisation des EA }}$

\begin{tabular}{|c|c|l|}
\hline \multirow{2}{*}{$\begin{array}{l}\text { Facteurs } \\
\text { Externes }\end{array}$} & Marché & Taille du marché domestique (niche ou non) \\
\cline { 2 - 3 } & Profil des Clients & Degré d'internationalisation des clients domestiques (B2B) \\
\cline { 2 - 3 } & Réseaux & Insertion dans des réseaux au démarrage \\
\hline \multirow{3}{*}{$\begin{array}{l}\text { Facteurs } \\
\text { Internes }\end{array}$} & Entreprise & $\begin{array}{l}\text { Degré de rareté du savoir-faire } \\
\text { Degré d'innovation des produits/services }\end{array}$ \\
\cline { 2 - 3 } & Dirigeant & $\begin{array}{l}\text { Compétences à l'international (linguistiques, techniques, } \\
\text { culturelles...) } \\
\text { Orientation entrepreneuriale }\end{array}$ \\
\hline
\end{tabular}

La recension de ces différents facteurs potentiels d'influence débouche sur notre seconde question de recherche : quels sont les facteurs explicatifs du processus d'internationalisation des EA ?

\section{3/ UNE METHODOLOGIE QUALITATIVE FONDEE SUR SEPT ETUDES DE CAS}

Compte tenu de la quasi-inexistence des recherches sur le processus d'internationalisation des entreprises artisanales, la complexité du phénomène et la nécessité de démêler leurs dynamiques sous-jacentes (Yin, 1984 ; Eisenhardt, 1989 ; Siggelkow, 2007), nous avons opté pour une démarche qualitative fondée sur des études de cas réalisées de façon rétrospective (Bell et al., 2003).

\footnotetext{
${ }^{6}$ L'orientation entrepreneuriale est ici définie comme l'inclinaison à rechercher et saisir des opportunités, à prendre des risques et à créer de la nouveauté (Wiklund et Sheperd, 2005 ; Fayolle, 2010 ; Chabaud et Messeghem, 2010), et ce, dans le cas qui nous intéresse ici, à l'international.
} 
En suivant les conseils d'Eisenhardt (1989), pour qui le nombre idéal de cas se situe entre quatre et dix, nous avons retenu sept cas d'entreprises artisanales figurant sur des listes d'entreprises exportatrices de Chambres de Métiers et de l'Artisanat que nous avons contactées pour l'étude. Ces entreprises ont été choisies parce qu'elles réalisent plus de 10\% de leur CA à l'export et parce qu'elles exportent leurs produits dans des zones géographiques variées, et pas seulement en Europe ou dans des pays francophones et frontaliers. Bien qu'appartenant toutes au Répertoire des Métiers, ces entreprises ont également été sélectionnées parce qu'elles avaient des métiers distincts, et ce afin d'introduire une certaine variété dans l'échantillon (Huberman et Miles, 1991). Elles appartiennent ainsi aux quatre secteurs principaux de l'artisanat que sont l'alimentation, le bâtiment, la production et les services. Leur taille est également différente, même si elle respecte les limites généralement admises pour les EA (entre 0 et 20 salariés). Leur C.A à l'export varie enfin fortement (de 10 à 90\%). Les principales caractéristiques de ces entreprises sont présentées dans le tableau $\mathrm{n}^{\circ} 3$. Le recueil des données s'est principalement appuyé sur des entretiens réalisés avec les dirigeants de ces entreprises artisanales et, quand ce poste existait, le responsable export. Deux à trois entretiens ont été réalisés pour chaque entreprise, d'une durée assez longue ( $3 \mathrm{~h}$ en moyenne $)^{7}$. Afin de limiter certains biais dus à l'étude rétrospective des processus, des données secondaires ont été également été analysées (documents commerciaux comme les plaquettes en langues étrangères, les sites web, les contrats avec les agents/distributeurs et des documents financiers). Une visite de chaque atelier a aussi eu lieu pour mieux comprendre le contexte des EA : les produits et services commercialisés, l'organisation du travail dans l'atelier et dans la fonction commerciale, le type de rapport entretenu avec les clients au quotidien...

Pour coder les données, nous avons utilisé une procédure d'analyse de contenu (Strauss, 1987). Pour l'analyse intra-site, nous avons codé les données en thèmes correspondant aux différentes composantes de notre cadre d'analyse : rapidité et précocité du processus et facteurs explicatifs (cf. tableaux $\mathrm{n}^{\circ} 1$ et 2 ). Nous avons ainsi pu caractériser le processus d'internationalisation suivi par chaque cas. Nous avons ensuite procédé à une analyse transversale pour faire émerger des patterns et tenter de comprendre les facteurs expliquant la nature de ce processus, en utilisant des matrices destinées à faciliter la comparaison des sept cas (Huberman et Miles, 2003).

\footnotetext{
${ }^{7}$ L'auteur remercie Christian PICARD (IREGE) pour la réalisation de deux de ces entretiens.
} 
Tableau n³ : caractéristiques de l'échantillon étudié

\begin{tabular}{|c|c|c|c|c|c|c|c|}
\hline & Machinalim & Peinturmat & Luther & Traçalim & Transporméca & Mécano & Ebène \\
\hline Activité & $\begin{array}{l}\text { Produits et services pour } \\
\text { restaurateurs }\end{array}$ & $\begin{array}{l}\text { Vente à distance de } \\
\text { matériel de } \\
\text { peinture }\end{array}$ & $\begin{array}{l}\text { Luthier (fabrication, } \\
\text { réparation et } \\
\text { restauration } \\
\text { d'instruments de } \\
\text { musique) et } \\
\text { Archeterie }\end{array}$ & $\begin{array}{c}\text { Fabrication } \\
\text { d'identifiants pour la } \\
\text { traçabilité } \\
\text { alimentaire }\end{array}$ & $\begin{array}{l}\text { Fabrication de } \\
\text { convoyeurs pour } \\
\text { transporter les } \\
\text { plateaux repas }\end{array}$ & $\begin{array}{c}\text { Fabrication } \\
\text { d'installations } \\
\text { automatisées de } \\
\text { lignes de } \\
\text { production }\end{array}$ & Ebénisterie de luxe \\
\hline Date de création & 1998 & 1998 & 1996 & $\begin{array}{c}1936 \\
\text { Reprise en } 2004 \\
\end{array}$ & $\begin{array}{c}1973 \\
\text { Reprise en } 1988 \\
\end{array}$ & 2005 & $\begin{array}{c}1885 \\
\text { Reprise en } 2009 \\
\end{array}$ \\
\hline $\begin{array}{c}\text { Date début } \\
\text { internationalisation }\end{array}$ & 1998 & 2003 & $\begin{array}{l}1996 \text { : les premiers } \\
\text { clients étaient } \\
\text { étrangers }\end{array}$ & $\begin{array}{c}\mathrm{Nd} \\
\text { (précédent } \\
\text { propriétaire) }\end{array}$ & $\begin{array}{l}1994 \text { (définition } \\
\text { d'une stratégie } \\
\text { export) }\end{array}$ & 2008 & Années 1960 \\
\hline Clientèle & $\begin{array}{l}\text { Particuliers, } \\
\text { collectivités, } \\
\text { entreprises }\end{array}$ & Particuliers & $\begin{array}{c}\text { Musiciens } \\
\text { professionnels et } \\
\text { amateurs, autres } \\
\text { luthiers, orchestres, } \\
\text { collectionneurs }\end{array}$ & $\begin{array}{l}\text { Industriels de } \\
\text { l'agro-alimentaire }\end{array}$ & $\begin{array}{c}\text { Restauration } \\
\text { collective (entreprises } \\
\text { de restauration, } \\
\text { écoles, hôpitaux, } \\
\text { armée...) }\end{array}$ & $\begin{array}{l}\text { Industriels des } \\
\text { secteurs de } \\
\text { l'automobile, du } \\
\text { bois et de } \\
\text { l'alimentaire }\end{array}$ & $\begin{array}{c}\text { Particuliers à pouvoir } \\
\text { d'achat élevés, décorateurs } \\
\text { (prescripteurs) }\end{array}$ \\
\hline CA (€) & 850000 & 700000 & 330000 & 1200000 & 2402000 & 4051100 & 9000000 \\
\hline $\begin{array}{l}\text { Part de l'export les } \\
\text { trois 1ères années }\end{array}$ & $30 \%$ & 0 & $50 \%$ & $\begin{array}{c}0 \\
\text { (30\% à la reprise) } \\
\end{array}$ & 0 & $10 \%$ & 0 \\
\hline Part actuelle export & $60 \%$ & $10 \%$ & $10 \%$ & $50 \%$ & $20 \%$ & $10 \%$ & $90 \%$ \\
\hline Nb salariés & 4 & 6 & 1 & 9 & 12 & 18 & 20 \\
\hline $\begin{array}{l}\text { Pays dans lesquels } \\
\text { l'export a démarré }\end{array}$ & $\begin{array}{c}\text { Grande Bretagne et } \\
\text { USA }\end{array}$ & Suisse, Allemagne & $\begin{array}{c}\text { Allemagne, Canada } \\
\text { et Japon }\end{array}$ & $\begin{array}{c}\text { Suisse, Italie, } \\
\text { Espagne }\end{array}$ & $\begin{array}{c}\text { Chine, Singapour, } \\
\text { Espagne }\end{array}$ & Mexique, Chine & $\begin{array}{c}\text { Etats-Unis, Italie, } \\
\text { Allemagne }\end{array}$ \\
\hline $\begin{array}{l}\text { Pays dans lesquels } \\
\text { l'EA commercialise } \\
\text { aujourd'hui ses } \\
\text { produits }\end{array}$ & $\begin{array}{l}60 \text { pays (Europe, Asie, } \\
\text { Moyen Orient, Etats- } \\
\text { Unis, Amérique du Sud, } \\
\text { Afrique) }\end{array}$ & $\begin{array}{l}\text { Europe, Amérique } \\
\text { du Sud, Afrique, } \\
\text { Moyen orient, } \\
\text { Europe de l'est, } \\
\text { Chine }\end{array}$ & $\begin{array}{l}\text { Allemagne, Etats- } \\
\text { Unis, Canada, } \\
\text { Japon, Italie et } \\
\text { Suisse }\end{array}$ & $\begin{array}{l}\text { Europe, Russie, } \\
\text { Pays de l'est, } \\
\text { Canada, Etats-Unis, } \\
\text { Amérique du Sud } \\
\text { (Chili, Brésil) }\end{array}$ & $\begin{array}{l}\text { Europe, M. Orient, } \\
\text { Afrique du Nord } \\
\text { (Maroc, Algérie), } \\
\text { Amérique du Sud } \\
\text { (Brésil, Chili), Chine }\end{array}$ & Mexique, Chine & $\begin{array}{c}\text { Etats-Unis, Europe (Italie, } \\
\text { Espagne, Allemagne, } \\
\text { Grande Bretagne, } \\
\text { Benelux), Russie, Moyen } \\
\text { Orient }\end{array}$ \\
\hline Pers. interrogées & Dirigeant + resp. export & Dirigeant & Les 2 dirigeants & Dirigeant & Responsable export & Dirigeant & Dirigeant \\
\hline
\end{tabular}




\section{LES TRAJECTOIRES D'INTERNATIONALISATION DES SEPT ENTREPRISES ARTISANALES ETUDIEES : QUELS ENSEIGNEMENTS ?}

Les résultats obtenus grâce à l'étude des sept cas d'entreprise artisanales permettent tout d'abord de mettre en évidence la variété des trajectoires d'internationalisation de ces entreprises (§3.1). Ils mettent également en exergue l'influence majeure jouée par les facteurs internes (compétences de l'entreprise et profil de son dirigeant) dans la nature et le déroulement de ces trajectoires (\$3.2). Ces résultats sont présentés puis discutés dans les paragraphes qui suivent.

\section{1 - UNE VARIETE DE TRAJECTOIRES D'INTERNATIONALISATION}

Le tableau 4 présente de façon synthétique les principales caractéristiques des trajectoires d'internationalisation suivis par les sept entreprises étudiées. Puis, le tableau 5 résume ces données dans la matrice des modèles types d'internationalisation. La lecture de ces tableaux nous permet tout d'abord de constater que près de la moitié des EA de l'échantillon ( 3 sur les 7 de l'échantillon) a effectivement suivi un processus de type «Uppsala 1 » (Johanson et Vahlne 1977), c'est-à-dire non instantanée et progressif, au moins dans un premier temps. Ces entreprises (Peinturmat, Traçalim 1et Ebène 1) ont démarré leur activité en France et ont cherché à exporter dans un second temps, le plus souvent sans réelle volonté stratégique, mais en réponse à la sollicitation d'un client (Peinturmat, Ebène 1). Leur développement international a alors été graduel, avec au départ des réponses à des clients plutôt francophones et proches géographiquement (faible distance psychique). Ensuite, devant le potentiel de développement que l'export pouvait représenter, elles ont formalisé la démarche : mise en place d'outils de communication (site Internet en plusieurs langues, pour toutes les entreprises), participation régulière à des salons internationaux (Peinturmat), voire création de salon (Peinturmat). La modalité d'internationalisation la plus souvent utilisée est le recours à des agents ou des distributeurs (Ebene 1, Traçalim1), certes par manque de connaissances des marchés et par manque de ressources, mais aussi du fait de la dispersion géographique des clients. Le modèle Uppsala (Johanson et Vahlne 1977), malgré les nombreuses critiques auxquelles il a été confronté (Meier et Meschi, 2010), semble donc bien toujours d'actualité pour certaines EA. 
Tableau ${ }^{\circ} 4$ : caractéristiques des processus d'internationalisation suivis par chaque entreprise

\begin{tabular}{|c|c|c|c|c|c|c|c|}
\hline & Machinalim & Peinturmat & Luther & Traçalim & Transporméca & Mécano & Ebène \\
\hline $\begin{array}{c}\text { Démarrage du } \\
\text { processus : } \\
\text { Précoce VS Non } \\
\text { instantanée }\end{array}$ & $\begin{array}{c}\text { Précoce } \\
\text { lers clients } \\
\text { internationaux obtenus } \\
\text { dès la 1ère année, à } \\
\text { l'occasion du salon } \\
\text { Equiphotel à Paris (15\% } \\
\text { de visiteurs étrangers) }\end{array}$ & $\begin{array}{c}\text { Non instantané } \\
\text { Clients étrangers } \\
\text { apparus au bout de } 5 \\
\text { ans }\end{array}$ & $\begin{array}{c}\text { Précoce } \\
\text { Sous-traitance pour } \\
\text { anciens patrons } \\
\text { étrangers + bénéfice } \\
\text { de la localisation } \\
\text { dans une région } \\
\text { frontalière. }\end{array}$ & $\begin{array}{c}\text { Non instantané } \\
\text { Activité domestique } \\
\text { les premières années }\end{array}$ & $\begin{array}{c}\text { Non instantané } \\
\text { Export envisagé } \\
\text { seulement en } 1994 \\
\text { pour faire face à une } \\
\text { crise importante sur } \\
\text { le marché français et } \\
\text { assurer la pérennité } \\
\text { de l'entreprise }\end{array}$ & $\begin{array}{c}\text { Non instantané } \\
\text { Activité domestique } \\
\text { les premières années. } \\
\text { Internationalisation } \\
\text { démarrée pour suivre } \\
\text { les clients français }\end{array}$ & $\begin{array}{l}\text { Non instantané } \\
\text { Activité domestique } \\
\text { les premières années } \\
\text { mais clientèle du } \\
\text { luxe internationale } \\
\text { (clients étrangers à la } \\
\text { recherche du savoir- } \\
\text { faire français) }\end{array}$ \\
\hline $\begin{array}{c}\text { Nature du } \\
\text { processus : } \\
\text { progressif VS } \\
\text { rapide } \\
\text { Vitesse de } \\
\text { progression du } \\
\text { CA export } \\
\text { Proximité des } \\
\text { pays } \\
\text { Modalité } \\
\text { d'engagement }\end{array}$ & $\begin{array}{c}\text { Très rapide } \\
\text { Croissance rapide du CA } \\
\text { export } \\
\text { Pays très éloignés (ex : } \\
\text { Thaïlande) } \\
\text { Export puis filiales de } \\
\text { production en Asie et aux } \\
\text { Etats-Unis }\end{array}$ & $\begin{array}{c}\text { Progressif } \\
\text { Export direct via site } \\
\text { Internet }\end{array}$ & $\begin{array}{c}\text { Progressif. } \\
\text { Volonté de } \\
\text { développer l'export } \\
\text { mais manque de } \\
\text { temps et de capacité } \\
\text { de production. } \\
\text { Pays pouvant être } \\
\text { éloignés (via des } \\
\text { réseaux). Export } \\
\text { direct ou sous- } \\
\text { traitance }\end{array}$ & $\begin{array}{c}\text { Internat }{ }^{\circ} \text { progressive } \\
\text { réalisée par le } \\
\text { précédent } \\
\text { propriétaire (30\% du } \\
\text { CA à la reprise). Puis } \\
\text { accélération à la } \\
\text { reprise. Pays auj. } \\
\text { lointains. Export via } \\
\text { distributeurs } \\
\text { obligatoire } \\
\text { (éparpillement géo } \\
\text { des petits clients) }\end{array}$ & $\begin{array}{c}\text { Rapide } \\
\text { en termes de CA et } \\
\text { en termes de zones } \\
\text { géographiques } \\
\text { Travail avec des } \\
\text { agents commerciaux } \\
\text { et distributeurs ou } \\
\text { exporte directement. }\end{array}$ & $\begin{array}{c}\text { Rapide } \\
\text { Progressif en termes } \\
\text { de CA } \\
\text { Mais modalité } \\
\text { d'entrée audacieuse } \\
\text { et pays visés éloignés } \\
\text { (joint-venture avec } \\
\text { sous-traitants en } \\
\text { Chine et au Mexique) }\end{array}$ & $\begin{array}{c}\text { Progressif } \\
\text { Constitution d'un } \\
\text { réseau d'agents } \\
\text { commerciaux } \\
\text { (50\% du CA à la } \\
\text { reprise). Puis } \\
\text { accélération à la } \\
\text { reprise. Suppression } \\
\text { des agents et } \\
\text { installation de } \\
\text { showrooms }\end{array}$ \\
\hline $\begin{array}{l}\text { Caractéristiques } \\
\text { du processus : }\end{array}$ & $\begin{array}{c}\text { Proactif. Participation } \\
\text { annuelle à des salons. Les }\end{array}$ & $\begin{array}{l}\text { Au départ réactif, } \\
\text { réponse aux }\end{array}$ & $\begin{array}{l}\text { Réactif. Les clients } \\
\text { étrangers se }\end{array}$ & $\begin{array}{l}\text { Proactif à la reprise : } \\
\text { gros investissement }\end{array}$ & $\begin{array}{l}\text { Proactif. De } 1994 \text { à } \\
1999 \text { : présence sur }\end{array}$ & $\begin{array}{l}\text { Au départ réactif: } \\
\text { suivi de deux clients }\end{array}$ & $\begin{array}{l}\text { Réactif à l'origine, } \\
\text { plus proactif depuis }\end{array}$ \\
\hline
\end{tabular}


XXVIe Conférence Internationale de Management Stratégique

\begin{tabular}{|c|c|c|c|c|c|c|c|}
\hline $\begin{array}{c}\text { Proactivité VS } \\
\text { réactivité ; degré } \\
\text { de formalisation } \\
\text { de la démarche } \\
\text { export }\end{array}$ & $\begin{array}{l}\text { clients viennent ensuite } \\
\text { d'eux-mêmes, soit par le } \\
\text { biais des salons, soit } \\
\text { grâce au site Internet } \\
\text { (l'ent. a été précurseur } \\
\text { dans l'utilisation de cet } \\
\text { outil de communication). } \\
\text { Structuration à partir de } \\
2006 \text { (embauche d'un } \\
\text { responsable export) }\end{array}$ & $\begin{array}{c}\text { sollicitations des } \\
\text { clients via le site } \\
\text { Internet, puis } \\
\text { participation à des } \\
\text { salons à l'étranger et } \\
\text { création en } 2007 \\
\text { d'un salon } \\
\text { international en } \\
\text { France }\end{array}$ & $\begin{array}{c}\text { déplacent, soit parce } \\
\text { qu'ils résident près } \\
\text { de l'entreprise } \\
\text { (frontaliers), soit à } \\
\text { l'occasion de leur } \\
\text { voyage en Europe } \\
\text { (effet réputation). A } \\
\text { aussi bénéficié d'une } \\
\text { opération mise en } \\
\text { place par BPI France. } \\
\text { Pas de prospection } \\
\text { car capacité de } \\
\text { production saturée. } \\
\text { Pas de formalisation } \\
\text { de la démarche }\end{array}$ & $\begin{array}{c}\text { pour développer } \\
\text { l'export (prospection, } \\
\text { site Internet). Mais } \\
\text { au final, prospection } \\
\text { peu concluante. Les } \\
\text { distributeurs } \\
\text { contactent } \\
\text { directement } \\
\text { l'entreprise grâce à } \\
\text { son site Internet. Le } \\
\text { processus est } \\
\text { aujourd'hui très } \\
\text { structuré (contrats). }\end{array}$ & $\begin{array}{c}\text { de nombreux salons } \\
\text { et prospection de } \\
\text { distributeurs à } \\
\text { l'étranger. Depuis } \\
\text { exploitation de ces } \\
\text { contacts et } \\
\text { participation à de } \\
\text { nouveaux salons. } \\
\text { L'apprentissage a été } \\
\text { progressif. } \\
\text { Aujourd'hui, } \\
\text { pratique quotidienne. } \\
\text { Démarche structurée, } \\
\text { formalisée }\end{array}$ & $\begin{array}{c}\text { principaux à } \\
\text { l'étranger } \\
\text { Puis volonté de se } \\
\text { développer en Chine. } \\
\text { Obtention d'un prix } \\
\text { «export } 2013 \text { » qui a } \\
\text { permis de financer } \\
\text { une mission de } \\
\text { prospection en Chine } \\
\text { en décembre } 2015\end{array}$ & $\begin{array}{c}\text { la reprise : } \\
\text { prospection, } \\
\text { participation à des } \\
\text { salons, travail avec } \\
\text { agence de presse, } \\
\text { développement de, } \\
\text { showroom, site } \\
\text { internet (storytelling) }\end{array}$ \\
\hline
\end{tabular}


Toutefois d'autres entreprises de l'échantillon ont suivi des trajectoires d'internationalisation certes non instantanées, mais plus rapides (Ebene2, Traçalim2, Mecano et Transpormeca). Ainsi Ebene2 et Traçalim2, bien que mentionnées précédemment comme ayant suivi des processus progressifs, ont connu une accélération de leur processus d'internationalisation lors de l'arrivée de nouveaux dirigeants après la transmission de l'entreprise. Ainsi Ebene est passée de 50 à $90 \%$ du CA réalisé à l'export en 6 ans (le CA ayant lui-même doublé sur la même période) et Traçalim de 30 à $50 \%$, en 5 ans. Ces entreprises pourraient donc s'apparenter à ce que la littérature appelle des Born Again Global (Bell et al, 2003 ; Dominguez et Mayrhoffer, 2016), à la différence que le CA réalisé à l'export avant la reprise était déjà conséquent. Il ne s'agit donc pas d'une expansion internationale démarrée de zéro. Transpormeca, est quant à elle réellement partie de zéro, l'expansion internationale ayant été une question de survie, face à un marché français en crise et une baisse du chiffre d'affaires de $25 \%$. Quelques temps après leur arrivée, les nouveaux dirigeants ont ainsi embauché un responsable export chargé de développer fortement l'international, sur un laps de temps relativement court.

$\underline{\text { Tableau } \mathrm{n}^{\circ} 5 \text { : classement des cas dans la matrice des modèles d'internationalisation }}$

\begin{tabular}{|c|c|c|c|}
\cline { 3 - 3 } \multicolumn{2}{c|}{} & \multicolumn{2}{|c|}{ Démarrage du processus } \\
\cline { 2 - 3 } Nature du & Progressif / Séquentiel & Non instantané & Précoce \\
\hline \multirow{3}{*}{ processus } & & Peinturmat & Luther \\
& & Traçalim 1 & \\
\cline { 2 - 4 } & Rapide & Ebène 1 & Machinalim \\
& & Traçalim 2 & \\
& & Ebène 2 & \\
& & Mécano & \\
& & Transporméca & \\
\hline
\end{tabular}

On relève également dans cette catégorie, des modalités d'internationalisation plus ambitieuses. Ainsi depuis sa reprise, Ebene remplace les agents par des implantations commerciales locales -des showrooms- dans les métropoles stratégiques, afin de mieux mettre en valeur son savoir-faire et le style unique de ses produits. La fabrication reste néanmoins en

\footnotetext{
${ }^{8}$ Les entreprises Traçalim et Ebene ont été placées à deux endroits différents (1 et 2) pour matérialiser les différences de processus d'internationalisation suivis avant et après la reprise.
} 
France, au plus près du savoir-faire. De même, si pour Mecano la part que représente l'international aujourd'hui est peu importante (10\%), cette EA n'a pas hésité à attaquer d'emblée des pays très éloignés (Mexique et Chine) avec une modalité impliquante d'internationalisation : une joint-venture avec un partenaire étranger. Le savoir-faire mis à disposition du partenaire est cependant peu spécifique (fabrications de produits standards), les produits plus sophistiqués restant fabriqués en France.

On trouve aussi dans l'échantillon un cas d'EA que l'on peut qualifier de born global (Oviatt et McDougall, 1994). En effet, l'entreprise Machinalim a, dès la première année de son existence, commercialisé ses produits à l'étranger, grâce à la participation à un salon international en France qui reçoit un nombre important de visiteurs étrangers. Le caractère innovant de la solution qu'elle propose (équipement + ingrédients prêts à l'emploi) lui a en effet permis de séduire d'emblée des clients étrangers attirés par cette offre facile d'utilisation et qui s'adapte à tous les contextes culturels. Elle a également très tôt dû ouvrir des filiales de production en Asie et aux Etats-Unis pour approvisionner plus rapidement en matières premières ses clients dans ces zones géographiques et pour en contourner les contraintes juridico-fiscales (taxes et normes alimentaires). Les machines restent toutefois assemblées en France.

Enfin, on note l'existence d'un processus un peu particulier d'internationalisation, car à la fois précoce et progressif. C'est celui suivi par l'entreprise Luther qui a eu comme premiers clients des clients étrangers (avant d'avoir des clients domestiques). En effet les dirigeants de cette entreprise ont, lors de l'ouverture de leur atelier de fabrication, commencé par réaliser de la sous-traitance pour les entreprises étrangères pour lesquelles ils avaient travaillé auparavant en tant que salarié. Cependant, malgré cette opportunité de réseau, les dirigeants n'ont pas eu les ressources en temps pour développer plus avant leur stratégie d'expansion internationale. La part du CA à l'export a régressé au fur et à mesure du développement de l'activité domestique et stagne aujourd'hui à $10 \%$.

$\mathrm{Au}$ final, cette variété constatée de trajectoires d'internationalisation va donc à l'encontre de la vision traditionnelle encore dominante de l'entreprise artisanale qui considère que ces entreprises tendent à privilégier le statu quo et leur pérennité, bien loin derrière la croissance et le profit (Polge, 2008 ; Boldrini et al, 2011) et qu'elles demeurent encastrées dans leur environnement local et proche (Gumpert et Boyd, 1984 ; Jaouen, 2006 ; Loup, 2008 ; Torrès et Jaouen, 2008). Les EA sont tout à fait capables, à l'instar d'entreprises positionnées sur des 
secteurs plus high tech, d'adopter des processus rapides et précoces d'expansion internationale. Certes dans près de la moitié des cas (4 sur 7 ; Peinturmat, Luther, Mecano et Ebene 1), les démarches ont plutôt été réactives, les EA se contentant au départ de répondre aux sollicitations de clients étrangers (Laghzaoui, 2009; Arlotto et al, 2011), via la participation à un salon international en France ou via le site internet de l'entreprise. Mais rapidement, devant le succès de cette première opération, les dirigeants adoptent souvent une démarche plus proactive, l'international devenant une réelle option stratégique. En outre, pour quatre entreprises (Machinalim, Tracalim 2, Transpormeca et Ebene2), le comportement a été d'emblée fortement proactif. Ces entreprises ont ensuite progressivement structuré et formalisé leur démarche export (embauche d'un responsable export, définition de pays cibles à attaquer, identification de salons stratégiques auxquels il faut participer, installation de bureaux ou showrooms à l'étranger dans des zones clés).

\section{2 - LES FACTEURS EXPLICATIFS DES PROCESSUS D'INTERNATIONALISATION}

Le tableau $n^{\circ} 6$ détaille les facteurs internes et externes susceptibles d'avoir joué un rôle dans le déroulement du processus d'internationalisation des EA.

En ce qui concerne les facteurs externes, plus de la moitié des entreprise de l'échantillon sont positionnées sur des marchés de niche, soit parce que la clientèle est en soi très spécifique et peu nombreuse (amateurs de peinture sur porcelaine pour Peinturmat, entreprises fromagères pour Traçalim, restauration collective pour Transporméca), soit parce que le segment visé dans le marché est restreint (clientèle de luxe pour Ebene, musiciens professionnels pour Luther). Ce facteur joue donc bien comme un motif d'expansion internationale, comme cela a déjà été souligné pour d'autres types d'entreprises que les EA (Oakey, 1995 ; Keeble, et al, 1998).

En revanche, le fait de travailler avec des clients français qui soient eux même déjà internationalisés ne semblent pas avoir particulièrement facilité le développement international des entreprises de l'échantillon (sauf pour Mécainno qui a, au départ, suivi ses donneurs d'ordre français à l'étranger), contrairement à ce que certains chercheurs laissaient entendre (Holmlund et Kock, 1998 ; Martin et al, 1998 ; Ageron, 2001 ; Bourcieu et al, 2006 ; Barbat et Hlady-Rispal, 2006). La plupart des entreprises étudiées travaillent en effet soit pour des clients franco-français, soit pour des clients s'étant internationalisés, mais sans faire bénéficier leur fournisseur de leur stratégie, réseau ou compétences. Comme nous l'avons vu 
précédemment en revanche, ce sont souvent les clients étrangers qui démarchent directement les EA françaises.

De même, l'insertion initiale dans des réseaux ne semble pas avoir joué un rôle aussi important pour les entreprises de l'échantillon que ne le laisse entendre la littérature (Coviello et Munro, 1997 ; Chetty et al, 2000 ; Ellis, 2000 ; Johanson et Vahlne, 2009). La plupart des EA étudiées ne bénéficiaient pas de réseau au démarrage de leur internationalisation. Celui-ci s'est construit progressivement, au même rythme que l'expansion internationale. L'effet réseau a joué dans deux cas seulement : celui de Luther, qui a permis à l'entreprise d'avoir comme premiers clients les EA étrangères pour lesquelles ses dirigeants avaient travaillé durant leur parcours d'apprentissage du métier, et celui de Mecano, qui a démarré son internationalisation en suivant ses clients donneurs d'ordre à l'étranger et en bénéficiant de leur connaissance sur ces marchés. Dans ces deux cas, le choix des pays où réaliser l'expansion internationale a été opportuniste et réalisé en fonction de la localisation géographique des partenaires du réseau (Canada et Allemagne pour Luther; Mexique et Chine pour Mecano), comme indiqué par Johanson et Valhne (2009) dans la version revisitée du modèle Uppsala.

Au niveau des facteurs internes, et tout d'abord sur le profil du dirigeant de l'EA, le constat est que le manque de compétences et d'expérience à l'international ne semble pas être un frein à l'internationalisation de ces entreprises. En effet, dans cinq entreprises sur sept, le processus a démarré alors que le dirigeant ne possédait aucune connaissance sur l'export ou les marchés internationaux, ni compétences en management international. En revanche, cette absence d'expérience et de compétences a souvent été compensée par une forte orientation entrepreneuriale, qui a permis à ces dirigeants de se former «sur le tas » et d'apprendre très rapidement, y compris de leurs erreurs (Machinalim, Tracalim 2, Transporméca, Mecano), notamment sur les compétences transversales ou génériques nécessaires à l'expansion internationale. 
Tableau $\mathrm{n}^{\circ} 6$ : les facteurs déterminant le processus d'internationalisation des entreprises étudiées

\begin{tabular}{|c|c|c|c|c|c|c|c|}
\hline & Machinalim & Peinturmat & Luther & Traçalim & Transporméca & Mécano & Ebène \\
\hline Marché de niche & Oui & Oui & $\begin{array}{c}\text { Non pour } \mathrm{B} 2 \mathrm{C} \text {, oui } \\
\text { pour } \mathrm{B} 2 \mathrm{~B}\end{array}$ & $\begin{array}{l}\text { Oui ( } 3 \text { concurrents dans } \\
\text { le monde, } 1200 \text { clients } \\
\text { potentiels dans le } \\
\text { monde) }\end{array}$ & Oui & Non & $\begin{array}{c}\text { Oui pour la clientèle } \\
\text { visée (luxe) }\end{array}$ \\
\hline Type de clientèle & $\mathrm{B} 2 \mathrm{~B}$ & $\mathrm{~B} 2 \mathrm{C}$ & $\begin{array}{c}\text { B2C et B2B (musiciens } \\
\text { amateurs ou } \\
\text { professionnels) }\end{array}$ & $\begin{array}{l}\text { B2B (industriels de } \\
\text { l'agroalimentaire) }\end{array}$ & $\begin{array}{c}\text { B2B (restauration } \\
\text { collective) }\end{array}$ & $\mathrm{B} 2 \mathrm{~B}$ & $\begin{array}{l}\text { B2C de luxe, rôle des } \\
\text { prescripteurs } \\
\text { (décorateurs) }\end{array}$ \\
\hline $\begin{array}{c}\text { Rôle de } \\
\text { l'internationalisation } \\
\text { des clients français }\end{array}$ & $\begin{array}{l}\text { Modéré } \\
\text { Joue pour une partie de } \\
\text { l'activité (ex : clubs de } \\
\text { vacances, restauration } \\
\text { en franchises), mais } \\
\text { l'essentiel de la } \\
\text { clientèle est composée } \\
\text { d'entrepreneurs ou de } \\
\text { restaurateurs étrangers }\end{array}$ & Non & $\begin{array}{c}\text { Faible à modéré } \\
\text { Effet bouche à oreille } \\
\text { entre artistes dans les } \\
\text { orchestres et les } \\
\text { tournées internationales }\end{array}$ & $\begin{array}{c}\text { Modéré } \\
\text { Certains clients français } \\
\text { sont de grands groupes } \\
\text { internationaux qui } \\
\text { rachètent des } \\
\text { entreprises à l'étranger. } \\
\text { Cela ouvre alors des } \\
\text { marchés à l'entreprise. } \\
\text { Toutefois cela } \\
\text { n'impacte pas de façon } \\
\text { significative le } \\
\text { développement export } \\
\text { de l'entreprise }\end{array}$ & $\begin{array}{c}\text { Non } \\
\text { Minorité de clients } \\
\text { français } \\
\text { internationaux qui n'ont } \\
\text { pas joué un rôle clé } \\
\text { dans le processus } \\
\text { d'internationalisation }\end{array}$ & $\begin{array}{c}\text { Oui } \\
\text { Clients automobiles qui } \\
\text { partent ouvrir des } \\
\text { usines en Chine et au } \\
\text { Mexique = l'entreprise } \\
\text { décide de les suivre }\end{array}$ & Non \\
\hline $\begin{array}{c}\text { Appui sur des réseaux } \\
\text { au démarrage du } \\
\text { processus }\end{array}$ & Non & $\begin{array}{l}\text { Non (possède un réseau } \\
\text { de professeurs de } \\
\text { peinture et de peintres } \\
\text { étrangers) mais sans } \\
\text { lien avec l'export }\end{array}$ & Oui (anciens patrons) & Non & Non & $\begin{array}{l}\text { Oui : sous-traitance } \\
\text { pour clients donneurs } \\
\text { d'ordre internationaux }\end{array}$ & Non \\
\hline
\end{tabular}


XXVIe Conférence Internationale de Management Stratégique

\begin{tabular}{|c|c|c|c|c|c|c|c|}
\hline $\begin{array}{l}\text { Compétences du } \\
\text { dirigeant à } \\
\text { l'international } \\
\text { (linguistiques, } \\
\text { techniques, } \\
\text { culturelles...) }\end{array}$ & $\begin{array}{c}\text { Non } \\
\text { Aucune langue parlée } \\
\text { au départ, recrutement } \\
\text { d'un responsable export } \\
\text { ayant un BTS } \\
\text { commerce international }\end{array}$ & $\begin{array}{c}\text { Non } \\
\text { Des séjours à l'étranger } \\
\text { pour sa passion mais } \\
\text { pas de compétences à } \\
\text { l'international }\end{array}$ & $\begin{array}{c}\text { Oui } \\
\text { Six ans d'expérience } \\
\text { internationale } \\
\text { (apprentissage en } \\
\text { Belgique, Allemagne, } \\
\text { Canada). Connaissance } \\
\text { de la clientèle et de } \\
\text { concurrents pour } \\
\text { lesquels de la sous- } \\
\text { traitance est réalisée. }\end{array}$ & $\begin{array}{c}\text { Non } \\
\text { Anglais de base (+ } \\
\text { cours), pas } \\
\text { d'expérience } \\
\text { internationale préalable } \\
\text { Apprentissage } \\
\text { progressif } \\
\text { (autoformation du } \\
\text { dirigeant). }\end{array}$ & \begin{tabular}{|c} 
Non \\
Pas de compétences \\
spécifiques des \\
dirigeants. Directeur \\
export (BTS Commerce \\
international) qui \\
maîtrise trois langues \\
étrangères (anglais, \\
espagnol et portugais) \\
mais a appris le reste \\
« sur le tas»
\end{tabular} & $\begin{array}{c}\text { Non } \\
\text { Pas de compétence } \\
\text { internationale }\end{array}$ & $\begin{array}{c}30 \text { ans d'expérience } \\
\text { dans un groupe } \\
\text { international du } \\
\text { nouveau repreneur et de } \\
\text { sa femme }\end{array}$ \\
\hline $\begin{array}{c}\text { Orientation } \\
\text { entrepreneuriale du } \\
\text { dirigeant vers le } \\
\text { développement } \\
\text { international } \\
\text { (intensité de la recherche } \\
\text { d'opportunités, de la prise } \\
\text { de risque, et de l'aptitude } \\
\text { à la création de } \\
\text { nouveauté) }\end{array}$ & $\begin{array}{c}\text { Forte orientation vers le } \\
\text { développement } \\
\text { international en } \\
\text { saisissant toutes les } \\
\text { opportunités. Dirigeant } \\
\text { prêt à prendre des } \\
\text { risques. }\end{array}$ & Faible & $\begin{array}{l}\text { Faible. Des passionnés } \\
\text { par le savoir-faire avant } \\
\text { tout. }\end{array}$ & $\begin{array}{c}\text { Forte volonté de faire } \\
\text { croître l'entreprise par } \\
\text { l'innovation et l'export. } \\
\text { Recherche active de } \\
\text { débouchés à } \\
\text { l'international, y } \\
\text { compris dans des pays } \\
\text { pouvant être jugés } \\
\text { risqués. Mise au point } \\
\text { d'innovation permettant } \\
\text { l'internationalisation }\end{array}$ & $\begin{array}{c}\text { Forte volonté de faire } \\
\text { croître l'entreprise par } \\
\text { l'innovation et l'export. } \\
\text { Recherche active } \\
\text { d'opportunités à } \\
\text { l'international }\end{array}$ & $\begin{array}{l}\text { Forte. Dirigeant prêt à } \\
\text { prendre des risques } \\
\text { pour s'internationaliser. }\end{array}$ & $\begin{array}{c}\text { Forte. Le } \\
\text { développement de } \\
\text { l'entreprise passe par } \\
\text { l'international donc } \\
\text { volonté de saisir les } \\
\text { opportunités et de } \\
\text { s'engager activement } \\
\text { sur le smarchés. }\end{array}$ \\
\hline Rareté du savoir-faire & $\begin{array}{c}\text { Savoir-faire français } \\
\text { (l'aliment préparé est } \\
\text { par définition français) } \\
\text { Package de } \\
\text { produits/service qui }\end{array}$ & $\begin{array}{l}\text { Non } \\
\text { Mais certaine créativité }\end{array}$ & $\begin{array}{l}\text { Savoir-faire en lutherie } \\
\text { courant mais } \\
\text { spécialisation en haute } \\
\text { restauration rare (des } \\
\text { années d'apprentissage }\end{array}$ & $\begin{array}{c}\text { Savoir-faire rare ( } 3 \\
\text { concurrents dans cette } \\
\text { activité). Certification } \\
\text { Iso } 22000\end{array}$ & $\begin{array}{c}\text { Sur-mesure, grande } \\
\text { complexité technique } \\
\text { des installations } \\
\text { (parfois sous-traitants } \\
\text { de concurrents qui ne }\end{array}$ & Non & $\begin{array}{c}\text { Savoir-faire en } \\
\text { fabrication classique, } \\
\text { mais plus rare en ce qui } \\
\text { concerne les finitions } \\
\text { (originalité du style) }\end{array}$ \\
\hline
\end{tabular}

Lyon, 7-9 juin 2017 
XXVIe Conférence Internationale de Management Stratégique

\begin{tabular}{|c|c|c|c|c|c|c|c|}
\hline & $\begin{array}{c}\text { rend l'offre attractive et } \\
\text { rare } \\
\text { Normes internationales } \\
\text { d'hygiène }\end{array}$ & & $\begin{array}{l}\text { chez différents luthiers } \\
\text { et beaucoup } \\
\text { d'autoformation). } \\
\text { Réalisation de pièces } \\
\text { uniques sur-mesure } \\
\text { Savoir-faire en } \\
\text { archeterie plus rare (40 } \\
\text { ateliers en France) } \\
\text { Combinaison des deux } \\
\text { savoir-faire (archeterie } \\
+ \text { lutherie) encore plus } \\
\text { rare }\end{array}$ & & $\begin{array}{c}\text { maitrisent pas la } \\
\text { technicité de leur } \\
\text { savoir-faire). Seule } \\
\text { entreprise à avoir la } \\
\text { certification NF } \\
\text { hygiène alimentaire }\end{array}$ & & Modèles déposés \\
\hline $\begin{array}{l}\text { Degré d'innovation } \\
\text { des produits }\end{array}$ & $\begin{array}{c}\text { Elevé } \\
\text { Plusieurs brevets } \\
\text { déposés }\end{array}$ & Faible & $\begin{array}{l}\text { Savoir-faire au } \\
\text { contraire très } \\
\text { traditionnel, peu } \\
\quad \text { innovant }\end{array}$ & $\begin{array}{c}\text { Elevé } \\
\text { Incorporation } \\
\text { d'électronique dans des } \\
\text { produits au départ } \\
\text { traditionnels }\end{array}$ & $\begin{array}{c}\text { Elevé } \\
\text { Produits très innovants } \\
(30 \% \text { du temps } \\
\text { consacré à la } \mathrm{R} \& \mathrm{D}) ; \\
2 \text { à } 3 \text { produits nouveaux } \\
\text { par an }\end{array}$ & $\begin{array}{l}\text { Faible à l'origine mais } \\
\text { élevé aujourd'hui grâce } \\
\text { à la conception d'un } \\
\text { palettiseur robotisé à } \\
\text { haute performance } \\
\text { breveté }\end{array}$ & $\begin{array}{c}\text { Savoir-faire traditionne } \\
\text { mais rôle de la } \\
\text { créativité }\end{array}$ \\
\hline
\end{tabular}


Grâce à leur proactivité, certaines n'ont également pas hésité à s'entourer de personnes ayant les compétences nécessaires (embauche de responsables exports dans trois cas ou développement de réseaux d'agents commerciaux ou distributeurs possédant les compétences pays).

Parmi les autres facteurs internes facilitant l'internationalisation, on retrouve également la rareté du savoir-faire. Les EA de l'échantillon sont plutôt positionnées sur des marchés « hauts de gamme », qui font appel à la créativité au sens large (fabrication à la main, qualité de finition, respect des normes les plus strictes ou adaptation parfaite à des demandes complexes ; Luther ; Tracalim ; Ebene). Elles délivrent des prestations sur-mesure que peu de concurrents sont capables de reproduire (Transpormeca). La maîtrise de ce savoir-faire rare s'appuie sur une main d'œuvre très qualifiée et expérimentée et sur des équipements techniques modernes (parfois présentés comme les plus modernes du marché). Notons également qu'une caractéristique forte des entreprises positionnées dans des métiers d'art ou alimentaires est de revendiquer un savoir-faire «à la française », reconnu à l'étranger (Machinalim, Ebene). En définitive, les clients étrangers viennent à ces EA parce qu'elles détiennent un savoir-faire rare, parfois labélisé «Made in France », qui leur confère une certaine notoriété, leur fait bénéficier d'un bouche à oreille favorable, qu'ils sont capables de mettre en avant sur un site internet vitrine. Dans certains cas toutefois, ce savoir-faire peut s'avérer un handicap. C'est le cas pour Luther qui, de par le caractère manuel de la fabrication, se voit doté d'une capacité de production très limitée qui ne lui permet pas de développer davantage l'export.

Pour les entreprises qui manquent de savoir-faire spécifique, ce dernier peut être comblé par le caractère innovant des produits ou services proposés. C'est le cas de Machinalim, qui possède de nombreux brevets autour du package de produits/services que l'entreprise a élaboré, et aujourd'hui de Mecano, qui va se servir d'une innovation pour développer l'export de façon plus proactive, alors qu'au départ elle avait plutôt une position de suiveur de ses clients à l'international. Dans certains cas, le degré d'innovation complète un savoir-faire initial déjà spécifique (Tracalim, Transpormeca).

Nous avons ensuite cherché à savoir si derrière ces tendances explicatives générales, les différents facteurs pouvaient avoir une influence sur le déroulement (précocité et rapidité) des trajectoires d'internationalisation (tableau $\mathrm{n}^{\circ}$ ). A la lecture de ce tableau, il semblerait qu'un développement international rapide de l'EA repose à la fois sur les compétences de 
l'entreprise (savoir-faire rare et/ou capacité d'innovation) et sur l'orientation entrepreneuriale du dirigeant. Lorsque ces deux conditions internes ne sont pas réunies, le développement international semble plus progressif. La théorie fondée sur les ressources et les compétences (Resource Based View et Competence-Based View ; Wernerflet, 1984 ; Barney, 1991 ; Grant, 1991) permet donc en partie d'expliciter ce résultat. Le savoir-faire spécifique maîtrisé par une entreprise artisanale (Picard, 2006 ; Polge, 2008) et/ou le caractère innovant des solutions proposées (Oviatt et McDougal, 1994) semblent être à la base du déploiement de stratégies rapides d'expansion internationale. Cette recherche vient aussi corroborer les résultats des travaux qui soulignent le rôle de l'attitude du dirigeant et de ses capacités dans le cadre d'événements entrepreneuriaux comme l'expansion internationale (Coviello et Munro, 1995 ; Tseng et al, 2004 ; Dominguez et Mayrhofer, 2016). Dans nos cas, le profil du dirigeant ne semble pas tant influencer le fait de s'internationaliser, mais la rapidité avec lequel ce processus se déroule et les modalités qu'il emprunte.

Tableau $\mathrm{n}^{\circ} 7$ : le rôle des facteurs externes et internes dans les trajectoires $\underline{\text { d'internationalisation des EA }}$

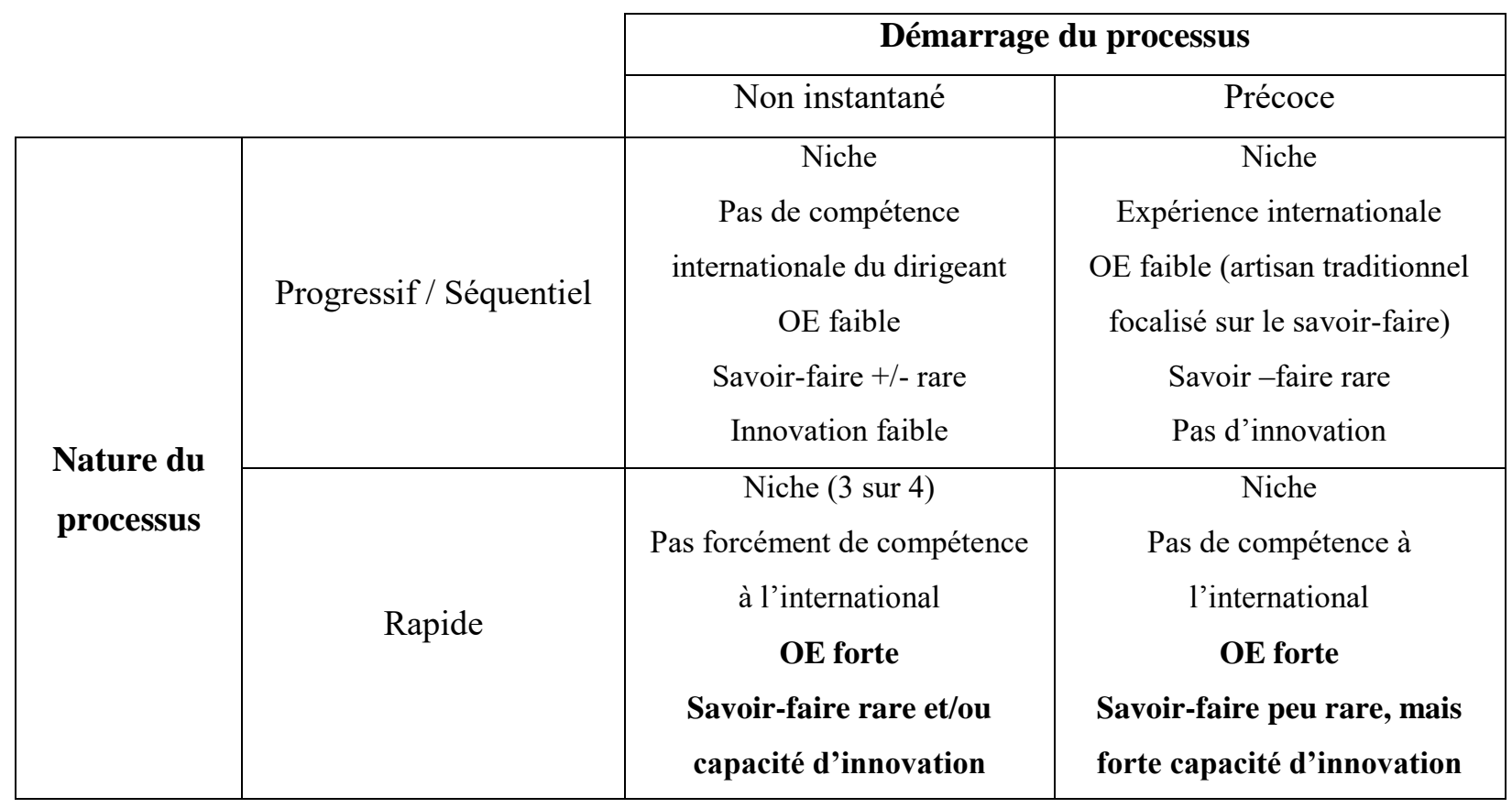




\section{CONCLUSION}

Ce travail de recherche exploratoire avait pour objectif d'enrichir les connaissances sur les trajectoires d'internationalisation des EA, en décrivant les formes qu'elles peuvent prendre, en termes de précocité et de rapidité, et en repérant les facteurs influençant ces deux dimensions du processus. Les sept études de cas réalisées ont permis de faire émerger deux principaux résultats.

Tout d'abord, cette recherche montre que l'internationalisation peut se manifester de différentes manières au sein d'une entreprise artisanale. Il n'existe pas de trajectoire type. Certes, certaines ont recours à des processus d'internationalisation à la fois tardif (qui succèdent à une première période pendant laquelle l'entreprise a privilégié son marché domestique) et progressif, comme ceux décrits par exemple par le modèle Uppsala (Johanson et Vahlne 1977). Mais on peut aussi trouver, au sein de ces entreprises souvent considérées comme traditionnelles et privilégiant la proximité dans les échanges, des trajectoires d'internationalisation précoces ou rapides, voire les deux (autrement dit des born global), comme dans les secteurs high tech (Bell, 1995 ; Jones, 1999 ; Coviello et Munro, 1997). Les EA doivent en effet, comme toute entreprise, s'adapter aux mouvements de fonds qui changent la donne sur les marchés (internationalisation de la concurrence, convergences des attentes des clients au niveau mondial, ouverture de marchés prometteurs à l'étranger, développement des TIC) et ce d'autant plus qu'elles sont positionnées sur des marchés de niche.

Cette recherche a également mis en lumière deux facteurs internes favorisant non seulement l'expansion internationale, mais surtout sa rapidité, voire sa précocité. Il s'agit, d'une part, des compétences de l'EA, s'exprimant en termes de rareté du savoir-faire possédé ou de capacité d'innovation, et, d'autre part, de l'orientation entrepreneuriale du dirigeant de l'EA. Si le savoir-faire a toujours été au cœur de l'EA (Picard, 2006 ; Polge, 2008), ces entreprises bénéficient également aujourd'hui de l'évolution des profils de certains de leurs dirigeants (études supérieures, expériences sectorielles plus variées avant de créer ou de reprendre une EA...) qui leur permet d'adopter des stratégies plus audacieuses.

D'un point de vue managérial, ce travail débouche sur plusieurs apports. Il permet de faire prendre conscience aux entreprises artisanales que l'internationalisation est possible et qu'elle peut prendre des chemins variés. Le manque de compétences à l'international ne doit pas apparaitre comme un frein, puisqu'il est tout à fait possible de démarrer un processus export avec un nombre très limité de connaissances. De même, il n'est pas nécessaire d'avoir des 
réseaux préalables pour débuter l'expansion internationale. Cette recherche insiste en revanche sur le rôle clé du savoir-faire, atout des EA, et notamment sur la reconnaissance à l'international du Made in France. Ce savoir-faire doit être mis en avant par des moyens de communication adéquats, au premier rang desquels le site Internet de l'entreprise, et présentés dans des salons professionnels internationaux, même si ceux-ci se déroulent en France dans un premier temps. Ces résultats peuvent également intéresser les acteurs institutionnels, comme les Chambres de Métiers et de l'Artisanat, soucieux d'accompagner au mieux les entreprises dans leur démarche d'internationalisation. Ils montrent notamment l'intérêt de démarches marketing et de communication destinées à mettre en valeur le savoir-faire possédé (type storytelling) et l'utilité des salons professionnels internationaux pour promouvoir le savoir-faire français.

Ce premier travail exploratoire présente cependant plusieurs limites inhérentes à la méthodologie utilisée. Il s'appuie en effet sur un échantillon de taille réduite -sept entreprisesqui incluait parfois une seule entreprise dans une catégorie de processus (ex : une seule born global). Il pourrait être intéressant de réaliser des recherches qualitatives complémentaires se focalisant seulement sur un des quatre processus identifiés. Cela ouvre également sur une piste de recherche qui mobiliserait une méthodologie quantitative confirmatoire se fondant sur les premiers résultats obtenus. Enfin, les processus ont été étudiés de façon rétrospective. Une analyse longitudinale pourrait permettre d'affiner les données recueillies sur les différentes étapes qui ont permis à l'EA de se développer.

\section{REFERENCES}

Ageron, B. (2001). La construction d'une capacité de réponse d'une PMI à l'international: une mise à l'épreuve des faits du modèle de Bilkey et Tesar. Revue internationale PME, 14(2).

Andersson, S., Gabrielsson, J., et Wictor, I. (2004). International activities in small firms: examining factors influencing the internationalization and export growth of small firms. Canadian Journal of Administrative Sciences, 21(1), 22-34.

Arlotto, J., Cyr, A., Meier, O., et Pacitto, J. C. (2011). Très petite entreprise et croissance: à la découverte d'un continent inexploré. Management et Avenir, (3), 16-36.

Barbat, V., Hlady Rispal, M., et Randerson, K. (2014). Disentangling the roles of international entrepreneurial orientation and networking in the internationalisation process of SESBs. International Journal of Entrepreneurship and Small Business, 23(3), 363-384. 
Beleska-Spasova, E., Glaister, K. W., et Stride, C. (2012). Resource determinants of strategy and performance: The case of British exporters. Journal of World business, 47(4), 635-647. Bell, J. (1995). The internationalization of small computer software firms: A further challenge to "stage" theories. European journal of marketing, 29(8), 60-75.

Bell, J., McNaughton, R., Young, S., et Crick, D. (2003). Towards an integrative model of small firm internationalisation. Journal of International Entrepreneurship, 1(4), 339-362.

Bloodgood, J. M., Sapienza, H. J., et Almeida, J. G. (1996). The internationalization of new high-potential US ventures: Antecedents and outcomes. Entrepreneurship: Theory and Practice, 20(4), 61-77.

Boldrini, J.C., Chéné, E., et Journé-Michel, H. (2011), " L'innovation des entreprises artisanales : Les effets de proximités », Revue Française de Gestion, 4(213), 25-41.

Burgel, O., et Murray, G. C. (2000). The international market entry choices of start-up companies in high-technology industries. Journal of International Marketing, 8(2), 33-62.

Cabrol, M., et Nlemvo, F. (2011). Le rôle de l'expérience de l'entrepreneur dans le niveau d'internationalisation des jeunes entreprises. Management et Avenir, (10), 38-56.

Cassiman, B., et Golovko, E. (2011). Innovation and internationalization through exports. Journal of International Business Studies, 42(1), 56-75.

Cavusgil, S. T., et Knight, G. (2015). The born global firm: An entrepreneurial and capabilities perspective on early and rapid internationalization. Journal of International Business Studies, 46(1), 3-16.

Chetty, S., et Campbell-Hunt, C. (2003). Paths to internationalisation among small-to medium-sized firms: a global versus regional approach. European Journal of Marketing, $37(5 / 6), 796-820$.

Coviello, N., et Munro, H. (1997). Network relationships and the internationalisation process of small software firms. International business review, 6(4), 361-386.

Dominguez, N., et Mayrhofer, U. (2016). «Il n'est jamais trop tard pour entreprendre»: l'internationalisation des born-again globals. Revue de l'Entrepreneuriat, 15(1), 61-80.

Eisenhardt, K.M., (1989), « Building Theories from Case Study Research », Academy of Management Review, 14 (4), p. 532-550.

Ellis, P. (2000). Social ties and foreign market entry. Journal of international business studies, 31(3), 443-469. 
Fernandez-Ortiz, R., et Lombardo, G. F. (2009). Influence of the capacities of top management on the internationalization of SMEs. Entrepreneurship and Regional Development, 21(2), 131-154.

Holmlund, M., et Kock, S. (1998). Relationships and the internationalisation of Finnish small and medium-sized companies. International Small Business Journal, 16(4), 46-63.

Huberman, A.M. et Miles, M.B., (1991), Analyse des données qualitatives, Bruxelles, De Boeck Université.

Hutchinson, K., Quinn, B., et Alexander, N. (2006). The role of management characteristics in the internationalisation of SMEs: Evidence from the UK retail sector. Journal of small business and enterprise development, 13(4), 513-534.

Institut Supérieur des Métiers (2010), Les nouveaux entrepreneurs de l'artisanat de production. Caractéristiques et besoins d'accompagnement, juin.

Institut Supérieur des Métiers (2014), Tableau économique de l'artisanat, Collection Etudes et Recherches de l'ISM.

Jaeger, C., (2009), «L'artisanat, des territoires mouvants pour des choix personnels », dans Traité de l'artisanat et de la petite entreprise (p. 149-162), S. Boutillier, M. David et C. Fournier, Editions Educaweb.

Jaouen, A. (2006), «Les stratégies d'alliance des TPE artisanales », Revue Internationale $P M E$, vol. 19, n³-4, p. 111-136.

Jaouen A et O. Torrès (2008), Les TPE un management de proximité, Lavoisier, Paris, Jean-Amans, C., et Abdellatif, M. (2013). L'influence du risque pays et de la distance culturelle sur les modes de présence et de contrôle des PME à l'étranger. Management international, 18(1), 73-86.

Johanson, J., et Vahlne, J. E. (1977). The internationalization process of the firm-a model of knowledge development and increasing foreign market commitments. Journal of international business studies, 8(1), 23-32.

Johanson, J., et Vahlne, J. E. (1990). The mechanism of internationalisation. International marketing review, 7(4).

Johanson, J., et Vahlne, J. E. (2009). The Uppsala internationalization process model revisited: From liability of foreignness to liability of outsidership. Journal of international business studies, 40(9), 1411-1431. 
Jones, M. V. (1999). The internationalization of small high-technology firms. Journal of International marketing, 15-41.

Jones, M. V., Coviello, N., et Tang, Y. K. (2011). International entrepreneurship research (1989-2009): a domain ontology and thematic analysis. Journal of business venturing, 26(6), 632-659.

Keeble, D., Lawson, C., Smith, H. L., Moore, B., et Wilkinson, F. (1998). Internationalisation processes, networking and local embeddedness in technology-intensive small firms. Small Business Economics, 11(4), 327-342.

Laghzaoui, S. (2009). Internationalisation des PME: apports d'une analyse en termes de ressources et compétences. Management et Avenir, (2), 52-69.

Leonidou, L. C., et Katsikeas, C. S. (1996). The export development process: an integrative review of empirical models. Journal of international business studies, 27(3), 517-551.

Lin, K. H., et Chaney, I. (2007). The influence of domestic interfirm networks on the internationalization process of Taiwanese SMEs. Asia Pacific Business Review, 13(4), 565583.

Loup, S. (2008), « Proximité et actions collectives des TPE : illustration par le cas des métiers d'art », in A Jaouen et O. Torrès, Les TPE un management de proximité, Lavoisier, Paris, p.193-214.

Lu, J. W., et Beamish, P. W. (2001). The internationalization and performance of SMEs. Strategic management journal, 22(6-7), 565-586.

Madsen, T. K., et Servais, P. (1997). The internationalization of born globals: an evolutionary process?. International business review, 6(6), 561-583.

Majkgård, A., et Sharma, D. D. (1998). Client-following and market-seeking strategies in the internationalization of service firms. Journal of Business-to-Business Marketing, 4(3), 1-41.

Majocchi, A., Bacchiocchi, E., et Mayrhofer, U. (2005). Firm size, business experience and export intensity in SMEs: A longitudinal approach to complex relationships. International Business Review, 14(6), 719-738.

McDougall, P. P., Shane, S., et Oviatt, B. M. (1994). Explaining the formation of international new ventures: The limits of theories from international business research. Journal of business venturing, 9(6), 469-487.

McDougall, P. P., Oviatt, B. M., et Shrader, R. C. (2003). A comparison of international and domestic new ventures. Journal of international entrepreneurship, 1(1), 59-82. 
Meier, O., et Meschi, P. X. (2010). Approche Intégrée ou Partielle de l'Internationalisation des Firmes: Les Modèles Uppsala (1977 et 2009) face à l'Approche «International New Ventures» et aux Théories de la Firme. Management international, 15(1), 11.

Miesenbock, K. J. (1988). Small businesses and exporting: a literature review. International Small Business Journal, 6(2), 42-61.

Oakey, R. P. (1995). High-technology new firms. Paul Chapman Pub., Ltd..

Oviatt, B. M., et McDougall, P. P. (1994). Toward a theory of international new ventures. Journal of international business studies, 25(1), 45-64.

Oviatt, B.M., et McDougall, P.P. (2005). Defining international entrepreneurship and modeling the speed of internationalization. Entrepreneurship theory and practice, 29(5), 537554.

Orser, B.J., Hogard Scott, S. et Riding, A.L., (2000), " Performance, Firm size and management problem solving », Journal of Small Business Management, October, 42-58.

Pacitto, J.C., et Julien, P.A., (2006), «Le marketing est-il soluble dans la très petite entreprise ? », Revue Internationale des PME, 19(3-4), 78-110.

Paige, R.C. et M.A. Littrell (2002), «Craft retailers' criteria for success and associated business strategies », Journal of Small Business Management, vol. 40, n, p. 314-331

Paturel, R., et Richomme-Huet, K. (2007), « Le devenir de l'activité artisanale passe-t-il par l'activité entrepreneuriale? », Revue de l'Entrepreneuriat, 6(1), 29-52.

Pfeffer, J., et Salancik, G. R. (1978). The external control of organizations: A resource dependence approach. NY: Harper and Row Publishers.

Picard, C., (2006), « La représentation identitaire de la TPE artisanale », Revue Internationale PME, 19(3-4), 13-49.

Polge, M., (2008), «Diversité des entreprises artisanales en développement », Management et Avenir, 4(18), 133-147.

Rialp, A., Rialp, J., et Knight, G. A. (2005). The phenomenon of early internationalizing firms: what do we know after a decade (1993-2003) of scientific inquiry?. International business review, 14(2), 147-166.

Ruzzier, M., AntonciC, B., Hisrich, R. D., et Konecnik, M. (2007). Human capital and SME internationalization: A structural equation modeling study. Canadian Journal of Administrative Sciences, 24(1), 15-29. 
Servantie, V., Cabrol, M., Guieu, G., et Boissin, J. P. (2016). Is international entrepreneurship a field? A bibliometric analysis of the literature (1989-2015). Journal of International Entrepreneurship, 14(2), 168-212.

Siggelkow, N. (2007). Persuasion with case studies. Academy of management journal, 50(1), 20 .

Stanworth, J.et Curran, J., (1976), " Growth and the small firm : an alternative view », Journal of Management Studies, Mai, 13(2), 94-110.

Strauss, A., (1987), Qualitative analysis for social scientists, New York : Cambridge University Press.

Thévenard-Puthod, C., et Picard, C. (2015). Diversité des stratégies de croissance de l'entreprise artisanale et profil du dirigeant. Revue internationale PME, 28(3-4).

Torrès, O., (2003), «Petitesse des entreprises et grossissement des effets de proximité » Revue Française de Gestion, 144, mai-juin, 119-138.

Westhead, P., Wright, M., et Ucbasaran, D. (2001). The internationalization of new and small firms: A resource-based view. Journal of business venturing, 16(4), 333-358.

Wiklund, J. et Shepherd, D., (2003), «Aspiring for and achieving growth : the moderation role of resources and opportunities », Journal of Management Studies, 40(8), 1911-1941.

Zarca, B., (1986), L'artisanat français, du métier traditionnel au groupe social, Economica. Yin, R. (1994), Case study research: Design and methods (2nd ed.), Sage Publishing, Beverly Hills, CA. 